Article

\title{
Circulating Cell-Free DNA-Based Comprehensive Molecular Analysis of Biliary Tract Cancers Using Next-Generation Sequencing
}

\author{
Szilvia Lilla Csoma ${ }^{1}$, Judit Bedekovics ${ }^{1}$, Gergó Veres ${ }^{2} \mathbb{D}^{\text {, }}$, Anita Árokszállási ${ }^{3}$, Csilla András ${ }^{3}$, Gábor Méhes ${ }^{1}$ \\ and Attila Mokánszki ${ }^{1, * \mathbb{D}}$ \\ 1 Department of Pathology, Faculty of Medicine, University of Debrecen, H-4032 Debrecen, Hungary; \\ csoma.szilvia@med.unideb.hu (S.L.C.); bedekovics.judit@med.unideb.hu (J.B.); \\ gabor.mehes@med.unideb.hu (G.M.) \\ 2 Division of Radiology and Imaging Science, Department of Medical Imaging, Faculty of Medicine, \\ University of Debrecen, H-4032 Debrecen, Hungary; veres.gergo@med.unideb.hu \\ 3 Department of Oncology, Faculty of Medicine, University of Debrecen, H-4032 Debrecen, Hungary; \\ arokszallasi.anita@med.unideb.hu (A.Á.); andras.csilla@med.unideb.hu (C.A.) \\ * Correspondence: mokanszki.attila@med.unideb.hu; Tel.: +36-52-411-600
}

check for updates

Citation: Csoma, S.L.; Bedekovics, J.; Veres, G.; Árokszállási, A.; András, C.; Méhes, G.; Mokánszki, A. Circulating Cell-Free DNA-Based Comprehensive Molecular Analysis of Biliary Tract Cancers Using Next-Generation Sequencing. Cancers 2022, 14, 233. https://doi.org/ 10.3390/cancers 14010233

Academic Editor: Alessandro Granito

Received: 6 December 2021

Accepted: 1 January 2022

Published: 4 January 2022

Publisher's Note: MDPI stays neutral with regard to jurisdictional claims in published maps and institutional affiliations.

Copyright: (C) 2022 by the authors. Licensee MDPI, Basel, Switzerland. This article is an open access article distributed under the terms and conditions of the Creative Commons Attribution (CC BY) license (https:// creativecommons.org/licenses/by/ $4.0 /)$.
Simple Summary: In the era of personalized oncology, next-generation sequencing plays an important role in identifying mutations that may predict the molecular pathomechanism and manage biliary tract cancers (BTC) therapy. The peripheral blood of cancer patients represents variable amounts of cell-free DNA (cfDNA) released from the tumor. Tumor-derived cfDNA in BTCs also allows the effective monitoring of the molecular genetic profile and the response to chemotherapy. Our study aimed to identify genetic aberrations in cell-free and matched tumor DNA in BTCs. We assume that the efficacy of the LB-based sequencing provides a novel perspective for BTCs therapy.

\begin{abstract}
Biliary tract cancer (BTC) is a rare malignancy with a long disease course and an overall poor prognosis. Despite multiple chemotherapy agents, there is no defined second-line treatment opportunity for advanced BTCs. In the era of precision oncology, NGS plays an important role in identifying mutations that may predict the molecular pathomechanism and manage the BTC therapy. The peripheral blood liquid biopsy (LB) of cancer patients represents variable amounts of cell-free DNA (cfDNA) released from tumor foci of any anatomical location. Our study aimed to identify somatic mutations and tumor variant burden (TVB) in cell-free and matched tumor DNA. We found a positive correlation between the estimated tumor volume and cfDNA yield $(r=0.9326, p<0.0001)$. Comparing tissue and LB results, similar TVB was observed. SNVs were proven in $84 \%$ of the cases, while in two cases, only the LB sample was informative for molecular analysis. The most important aberrations in BTCs, such as FGFR2, IDH1, IDH2, KRAS, and TP53, could be detected in matched LB samples. Our prospective study demonstrates a minimally invasive testing approach to identify molecular genetic alterations in cholangiocarcinoma and gallbladder cancers. Clinical applications of cfDNA reflect by capturing the outstanding spatial tumor heterogeneity and guarantee novel aspects for the precision oncology treatment.
\end{abstract}

Keywords: biliary tract cancers; cholangiocarcinoma; liquid biopsy; cell-free DNA; estimated tumor volume; DNA yield; mutation profiling; next-generation sequencing (NGS)

\section{Introduction}

Biliary tract cancers (BTCs) are rare malignancies with an extended disease course with an inadequate prognosis and restricted oncotherapeutic options [1,2]. The epithelial cells from three distinct anatomic locations are responsible for BTC 's' transformation, resulting in three BTC subtypes, which are as follows: intrahepatic cholangiocarcinoma (IHCC), extrahepatic 
cholangiocarcinoma (EHCC), and gallbladder carcinoma (GBC). Over the past few decades, the prevalence and mortality ratio of BTCs have been increasing [3]. According to a recent study, the five-year survival rates for IHCC and EHCC are 12 and 30\%, respectively [3]. In clinical and histopathological views, the BTC subtypes have variable features, and they are often treated similarly $[4,5]$. Surgery is the curative approach; however, BTCs are often unresectable, gemcitabine chemotherapy and adjuvant with capecitabine remain the basic curative decision in BTCs treatment [6-8]. Gemcitabine was not effective in the PRODIGE 12 and BCAT trials $[9,10]$, in this case, second-line treatment is currently defined as FOLFOX based on the ABC-06 landmark trial [11]. Nowadays, some studies highlight immunotherapy's effectiveness in BTC management in cases with high expression of programmed death ligand-1 (PD-L1) and programmed death-1 (PD-1) [12-14].

Despite multiple chemotherapy agents, there is no accurate second-line treatment opportunity for advanced BTCs. The reason for the lack of therapeutic consensus is differences in underlying tumor etiology, and consequently, the three BTC subtypes have different molecular profiles [2,15-17]. The ideal solution is to find practical molecular genetic aberrations that may increase the oncological management of BTC cases. Nextgeneration sequencing (NGS) is a popular available technology with a wide spectrum of genes in an individual platform. In the era of personalized medicine, NGS plays an essential role in detecting and annotating aberrations that may predict the prognosis and management of BTC therapy. Despite this substantial approach, the raised difficulty of molecular factors promoting genetic variability in $\mathrm{GBC}$ requires a more accurate application of NGS to this malignancy [18].

The peripheral blood $(\mathrm{PB})$ of cancer patients contains varying and fluctuating amounts of cell-free DNA (cfDNA) emitted from tumor foci of any anatomical location. The principle of the minimally invasive liquid biopsy (LB) has been successfully transferred to clinical routine follow-up of solid tumors [19]. Cell-free nucleic acids spread at extremely low levels in the $\mathrm{PB}$, and consequently, detection of molecular genetic alterations demands high-throughput techniques, such as NGS. A small amount of literature data reflecting LB for BTC patients were published [20,21], although studying cfDNA has enormous clinical possibilities, such as in cases where it is difficult to carry out tissue biopsy (e.g., liver or gallbladder). PB sampling has minimal procedural risks, suitable for longitudinal follow-up of the patients, and with the help of LB, the effectiveness of the chemotherapy can be monitored.

The aims of our study were (i) to characterize histological and immunohistochemical (IHC) staining features of samples originating from the three subtypes of BTC patients, (ii) to quantify tumor-derived cfDNA, (iii) to find a correlation between estimated tumor volume (ETV) and nucleic acid concentration, (iv) to identify somatic mutations and tumor variant burden (TVB) in LB samples, (v) to compare alterations between histological and LB samples, and (vi) to elucidate genetic difference among the three subtypes of BTCs. For this purpose, histologic examination, IHC, ETV calculation, nucleic acid isolation from the two types of samples (tissue and LB), and NGS gene panel analysis targeting 67 genes (Archer VariantPlex Solid Tumor panel, Illumina MiSeq platform) were performed on all samples of 25 BTC patients.

\section{Materials and Methods}

\subsection{Patients Samples}

Altogether, 25 formaldehyde-fixed paraffin-embedded tissue (FFPE) and 25 paired PB LB BTC samples were tested. Because of the long disease course, LB was performed for the mean 24 months (range: 12-36) after initial tissue biopsy (IHCC and EHCC cases)/surgical resection (only GBC cases) to evaluate chemotherapy effectiveness. All protocols have been approved by the author's respective Institutional Review Board for human subjects (IRB reference number: 60355-2/2016/EKU and 4648-6/2018/EÜIG). 


\subsection{Tumor Volume Evaluation}

The tumor mass parameters have been determined by using computed tomography (CT) and/or magnetic resonance imaging (MRI) right before the initial tissue biopsy/resection. According to radiological practice, maximal tumor width (w) and length (l) were measured. Estimated tumor volume (ETV) has to be calculated using the following formula:

$$
\mathrm{V}\left(\mathrm{mm}^{3}\right)=\mathrm{w}^{2}\left(\mathrm{~mm}^{2}\right) \times 1(\mathrm{~mm}) \div 2
$$

\subsection{Histology and Immunohistochemistry}

Hematoxylin and eosin (H\&E) stainings were performed and carefully analyzed by pathology specialists. The samples containing at least $20 \%$ tumor cells were selected for DNA isolation. IHC of CK7 (clone OV-TL 12/30, 1:100 dilution, Dako, Agilent Technologies, Santa Clara, CA, USA), CK8/18 (clone 5D3, 1:100 dilution, Leica Biosystems, Wetzlar, Germany), CK19 (clone Ks19.1, 1:200 dilution, Biocare Medical, Pacheco, CA, USA), CK20 (clone Ks20.8, 1:200 dilution, Leica Biosystems, Wetzlar, Germany), CA19-9 (clone C241:5:1:4, 1:200 dilution, Leica Biosystems, Wetzlar, Germany), HSA (clone OCH1E5, 1:700 dilution, Dako, Agilent Technologies Company, Santa Clara, CA, USA), arginase (polyclonal, 1:20,000 dilution, Merck, Darmstadt, Germany), and glypican-3 (clone 1G12, 1:50 dilution, Biocare Medical, Pacheco, CA, USA) were performed to validate BTC diagnosis. PD-L1 IHC (clone SP142, 1:100 dilution, Abcam, Cambridge, UK) was carried out to study the possibility of immunotherapy.

\subsection{DNA Isolation}

QIAamp DNA FFPE Tissue Kit (Qiagen, Hilden, Germany) was applied for FFPE tissues genomic DNA (gDNA) extraction. The isolations were carried out according to the manufacturer's instructions and the gDNA was eluted in $50 \mu \mathrm{L}$ elution buffer.

EDTA anticoagulant blood samples were centrifuged at $3000 \times g$ for $10 \mathrm{~min}$. To eliminate cell residues, $5 \pm 0.1 \mathrm{~mL}$ plasma was spun down $(16,000 \times g, 10 \mathrm{~min})$. Cell-free DNA was extracted from PB plasma into $35 \mu \mathrm{L}$ elution buffer using QIAamp Circulating Nucleic Acid Kit (Qiagen, Hilden, Germany).

The DNA concentrations were measured in the Qubit dsDNA HS Assay Kit using a Qubit 4.0 Fluorometer (Thermo Fisher Scientific, Waltham, MA, USA).

DNA yield was calculated with the following formula:

DNA yield $(\mathrm{ng} / \mathrm{mL}$ plasma $)=$ DNA concentration $(\mathrm{ng} / \mu \mathrm{L}) \times$ elution volume $(35 \mu \mathrm{L}) /$ plasma volume $(\mathrm{mL})$.

\subsection{Next-Generation Sequencing}

The amplifiable DNA amounts were estimated according to the Archer PreSeq DNA Calculator Assay Protocol (Archer DX, Boulder, CO, USA). Libraries of the samples were constructed using Archer VariantPlex Solid Tumor Kit (Archer DX, Boulder, CO, USA). The final purified libraries were quantified with KAPA Universal Library Quantification Kit (Kapa Biosystems, Roche, Basel, Switzerland).

The indexed libraries were then submitted to Illumina MiSeq System (MiSeq Reagent kit v3 600 cycles, Illumina, San Diego, CA, USA). The libraries pooling, denaturation, and dilution were carried out according to the manufacturer's instructions. The terminating loading concentration was $8 \mathrm{pM}$ libraries and 1\% PhiX. Captured libraries were sequenced with a paired-end run to obtain $2 \times 150$ bp reads with at least $250 \times$ depth of coverage.

The fastq files were analyzed with the Local Virtual Machine application of Archer DX Analysis software (version 6.2.7; Archer DX, Boulder, CO, USA) for identifying singlenucleotide variants (SNVs) as well as insertions and deletions (indels). For the alignment, the human reference genome GRCh37 (equivalent UCSC version hg19) was used. The sequence quality was evaluated and the cutoff was determined to be $3 \%$ variant allele frequency (VAF) in FFPE samples and $2 \%$ in LB samples. Massive insertion/deletion (>50 bp) and compound structural changes could not be captured by the method. The 
results were defined using the latest version of the Human Genome Variation Society nomenclature. Individual gene aberrations were checked in the COSMIC (Catalogue of Somatic Mutations in Cancer), ClinVar, and TCGA (The Cancer Genome Atlas) databases for clinical significance. Single nucleotide polymorphisms (SNPs) were monitored using the dbSNP database. Detected pathogen variants were cross-checked in the OncoKB database for therapeutic options.

\subsection{Statistical Analysis}

Statistical analyses were performed with GraphPad Prism 9. Differences between the ETV and DNA concentration of the three subtypes of BTC patients were analyzed using an unpaired $t$-test. When comparing the average TVB of the three BTC subtypes, a nonparametric $t$-test was used. Association between the tumor volume and cfDNA concentration was examined with Pearson correlation test (Spearman's rho calculation). A value of $p<0.05$ was considered to be statistically significant.

\section{Results}

\subsection{Patients Clinicopathological Characteristics}

The clinicopathological features of the 25 BTC patients are shown in Table 1 . The average age was 64.7 (range: 43-80). The gender distribution was 13/12 male/female. According to the tumor localization, 15 IHCC, five EHCC, and five GBC cases were evolved. The average ETV was $265,589 \mathrm{~mm}^{3}$ with a broad range: $3179-1,230,187 \mathrm{~mm}^{3}$. In five cases, metastasis was present (case 2,3,7,13, and 14), the metastatic tumor volume was added to ETV. Tissue biopsy was performed in IHCC and EHCC cases, while resection was carried out only in potential operable GBC cases, as well. The oncological treatments included gemcitabine-based chemotherapy.

Table 1. Clinicopathological features of the BTC patients.

\begin{tabular}{|c|c|c|c|c|c|c|}
\hline Cases & Gender & Age (Years) & $\operatorname{ETV}\left(\mathrm{mm}^{3}\right)$ & Metastasis & BTC Subtype & Chemotherapy \\
\hline 1 & $\mathrm{~F}$ & 80 & 83,349 & no & IHCC & cisplatin plus gemcitabine, capecitabine, irinotecan \\
\hline 2 & $\mathrm{~F}$ & 75 & 88,200 & liver & IHCC & gemcitabine, bevacizumab \\
\hline 3 & M & 76 & 56,784 & liver & IHCC & capecitabine, cisplatin plus gemcitabin \\
\hline 4 & $\mathrm{M}$ & 59 & 37,044 & no & IHCC & cisplatin plus gemcitabine \\
\hline 5 & $\mathrm{M}$ & 68 & $1,680,000$ & no & IHCC & cisplatin plus gemcibatine, irinotecan, capecitabine \\
\hline 6 & M & 68 & 37,462 & no & IHCC & cisplatin plus gemcitabine, capecitabine \\
\hline 7 & M & 60 & 259,200 & peritoneum & IHCC & cisplatin plus gemcitabine, trametinib, everolimus \\
\hline 8 & $\mathrm{M}$ & 68 & 62,500 & no & IHCC & cisplatin plus gemcitabine \\
\hline 9 & $\mathrm{~F}$ & 63 & $1,230,187$ & no & IHCC & cisplatin plus gemcitabine \\
\hline 10 & $\mathrm{M}$ & 53 & 263,250 & no & IHCC & cisplatin plus gemcitabine, irinotecan \\
\hline 11 & $\mathrm{M}$ & 70 & 12,800 & no & IHCC & cisplatin plus gemcitabine, irinotecan, capeticabine \\
\hline 12 & $\mathrm{~F}$ & 61 & 283,500 & no & IHCC & cisplatin plus gemcitabine, capecitabine \\
\hline 13 & M & 75 & 9126 & liver & IHCC & capecitabine, gemcitabine \\
\hline 14 & $\mathrm{~F}$ & 49 & 140,625 & ovary & IHCC & cisplatin plus gemcitabine \\
\hline 15 & $\mathrm{~F}$ & 66 & 670,372 & no & IHCC & gemcitabine \\
\hline 16 & $\mathrm{~F}$ & 64 & 36,162 & no & EHCC & cisplatin plus gemcitabine, capecitabine \\
\hline 17 & M & 43 & 952,544 & no & EHCC & cisplatin plus gemcitabine, irinotecan \\
\hline 18 & M & 51 & 6664 & no & EHCC & cisplatin plus gemcitabine, nivolumab \\
\hline 19 & $\mathrm{~F}$ & 70 & 98,606 & no & EHCC & cisplatin plus gemcitabine \\
\hline 20 & $\mathrm{~F}$ & 71 & 32,000 & no & EHCC & cisplatin plus gemcitabine \\
\hline 21 & $\mathrm{~F}$ & 58 & 6750 & no & GBC & cisplatin plus gemcitabine, irinotecan \\
\hline 22 & M & 70 & 126,000 & no & GBC & cisplatin plus gemcitabine \\
\hline 23 & $\mathrm{M}$ & 68 & 3179 & no & GBC & cisplatin plus gemcitabine \\
\hline 24 & $\mathrm{~F}$ & 71 & 137,312 & no & GBC & cisplatin plus gemcitabine \\
\hline 25 & $\mathrm{~F}$ & 60 & 98,000 & no & GBC & capecitabine, cisplatin plus gemcitabine \\
\hline
\end{tabular}

The number represents the case ID. Estimated tumor volume (ETV) was calculated using the following formula: $\mathrm{V}\left(\mathrm{mm}^{3}\right)=\mathrm{w}^{2}\left(\mathrm{~mm}^{2}\right) \times 1(\mathrm{~mm}) \div 2$. BTC: biliary tract cancer, IHCC: intrahepatic cholangiocarcinoma, EHCC: extrahepatic cholangiocarcinoma, GBC: gallbladder carcinoma.

\subsection{Histological Features including Immunohistochemistry}

The tumor is composed of irregularly shaped, atypical glands with infiltrative growth. Viable tumor cells without necrosis were present in all samples. Tumor cells are pleo- 
morphic with a high nuclear/cytoplasmic ratio, marked atypia, and conspicuous nucleoli. Representative histological and immunohistochemical images are presented in Figure 1. IHC staining features of the patient samples are presented in Table 2. CK7 (24/25, 96\%), CK8/18 (23/25, 92\%), CK19 (25/25, 100\%), and CA19.9 (23/25, 92\%) positivity were found. CK20 (21/25, 84\%), HSA (25/25, 100\%), arginase $(22 / 25,88 \%)$, glypican $(23 / 25,92 \%)$, and PD-L1 (25/25, 100\%) immunostaining negativity were performed.

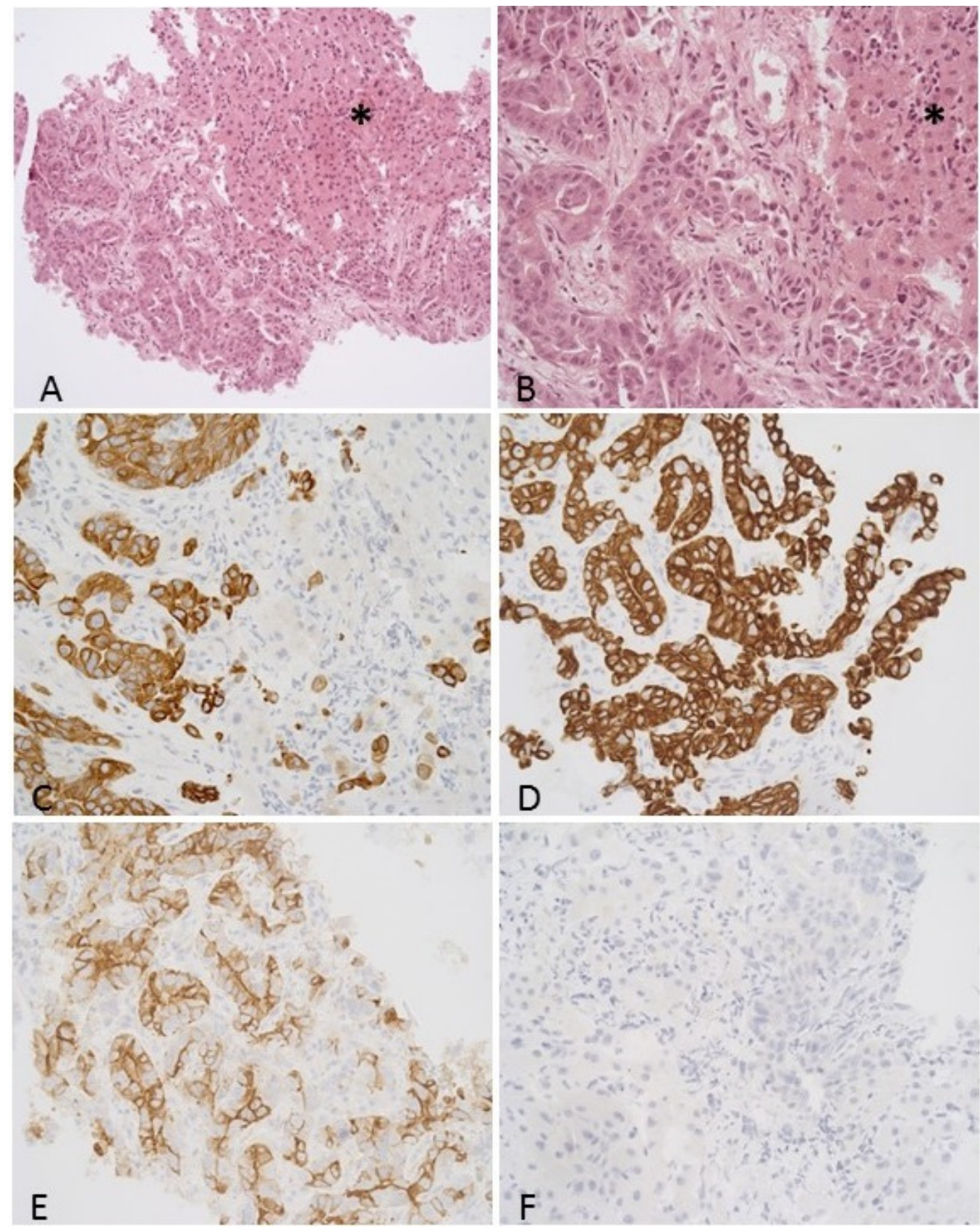

Figure 1. Representative histological and immunostaining of intrahepatic cholangiocarcinoma. (A,B): Core biopsy of a hepatic lesion which was consistent with intrahepatic cholangiocarcinoma (H\&E, 200× and $400 \times$ magnification, respectively). The tumor is made up of irregular, atypical glandules with infiltrative growth. The neoplastic glandules are surrounded by desmoplastic stroma. Non-neoplastic hepatocytes can be seen on the right side $\left(^{*}\right)$. The neoplastic cells were positive for CK7 (C), CK19 (D), and Ca19-9 (E), while negative for CK20 (F) immunostaining (400× magnification). 
Table 2. Immunohistochemical characteristics of the patient samples.

\begin{tabular}{|c|c|c|c|c|c|c|c|c|c|c|}
\hline Cases & Grading & CK7 & CK8/18 & CK19 & CK20 & CA19.9 & HSA & Arginase & Glypican & PD-L1 \\
\hline 1 & G2 & + & + & + & - & + & - & - & - & - \\
\hline 2 & G2 & + & + & + & + & + & - & - & - & - \\
\hline 3 & G3 & + & + & + & - & + & - & - & - & - \\
\hline 4 & $\mathrm{G} 2$ & + & + & + & - & + & - & - & - & - \\
\hline 5 & $\mathrm{G} 2$ & + & + & + & - & + & - & - & - & - \\
\hline 6 & $\mathrm{G} 2$ & + & - & + & - & + & - & - & - & - \\
\hline 7 & $\mathrm{G} 2$ & + & + & + & - & + & - & - & - & - \\
\hline 8 & G2 & + & + & + & - & + & - & - & $+^{*}$ & - \\
\hline 9 & G2 & + & + & + & + & + & - & - & - & - \\
\hline 10 & $\mathrm{G} 2$ & + & + & + & - & + & - & - & - & - \\
\hline 11 & G2 & + & + & + & - & + & - & + & - & - \\
\hline 12 & G3 & + & + & + & + & + & - & - & - & - \\
\hline 13 & $\mathrm{G} 2$ & + & + & + & - & - & - & - & - & - \\
\hline 14 & G3 & + & + & + & - & + & - & - & $+^{*}$ & - \\
\hline 15 & $\mathrm{G} 2$ & + & - & + & - & + & - & - & - & - \\
\hline 16 & G1 & + & + & + & - & + & - & - & - & - \\
\hline 17 & G3 & + & + & + & - & + & - & - & - & - \\
\hline 18 & $\mathrm{G} 2$ & - & + & + & - & + & - & - & - & - \\
\hline 19 & $\mathrm{G} 2$ & + & + & + & - & - & - & - & - & - \\
\hline 20 & G2 & + & + & + & - & + & - & - & - & - \\
\hline 21 & G2 & + & + & + & - & + & - & - & - & - \\
\hline 22 & $\mathrm{G} 2$ & + & + & + & + & + & - & - & - & - \\
\hline 23 & G3 & + & + & + & - & + & - & + & - & - \\
\hline 24 & G3 & + & + & + & - & + & - & + & - & - \\
\hline 25 & G2 & + & + & + & - & + & - & - & - & - \\
\hline
\end{tabular}

CK7: cytokeratin 7, CK8/18: cytokeratin 8/cytokeratin 18, CK19: cytokeratin 19, CK20: cytokeratin 20, CA19.9: cancer antigen 19-9, HSA: hepatocyte-specific antigen, PD-L1: programmed death-ligand 1 . $^{*}$ focal positivity.

\subsection{Correlation Analysis between Estimated Tumor Volume and cfDNA Yield}

No statistically significant differences were found in the ETV between the IHCC and the EHCC group ( $p=0.9928)$, between the IHCC and the GBC subtypes $(p=0.3192)$, and between the EHCC and the GBC category $(p=0.4376)$, when GBC considered a different entity, as well.

The mean cfDNA yield was $35.4 \mathrm{ng} / \mathrm{mL}$ plasma (range: 6.18-98.9). No statistically significant differences were found in the cfDNA yield between the IHCC and the EHCC $(p=0.7736)$, between the IHCC and the GBC $(p=0.1121)$, and between the EHCC and the GBC entity $(p=0.3570)$.

We found a positive significant correlation between the ETV and cfDNA yield using the Pearson correlation test $(r=0.9326, p<0.0001)$ analyzing all BTC cases (Figure 2).

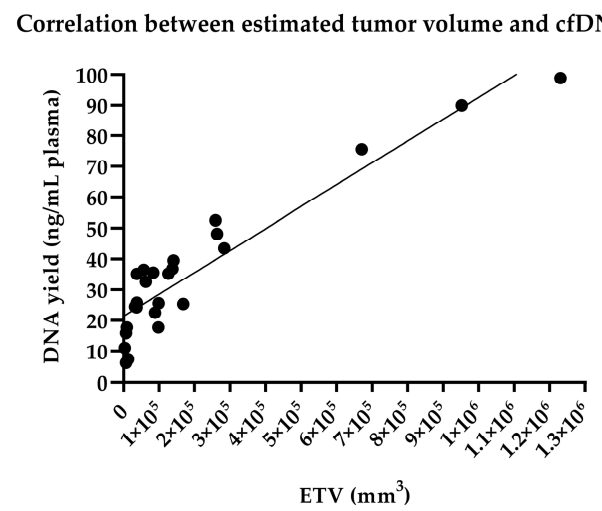

Figure 2. Association between estimated tumor volume (ETV) and tumor-derived circulating cell-free DNA yield. Pearson correlation coefficient was showed a significant correlation $(r=0.9326, p<0.0001$, 95\% confidence interval: 0.8510 to 0.9702 ). 


\subsection{NGS-Based Mutation Profiling of Genomic and Cell-Free DNA}

TVB was defined with the number of gene variants including SNPs above $2 \%$ VAF (Figure 3). TVB was calculated in tissue and liquid biopsy samples, as well. The largest TVB was in cases 2, 18, and 23, while no nucleotide change was detected in cases 1,17 , and 19. In cases 12 and 20, only the liquid biopsy was informative for the aspect of tumor burden determination. Comparing tissue and LB results, similar TVB was observed in most of the cases.

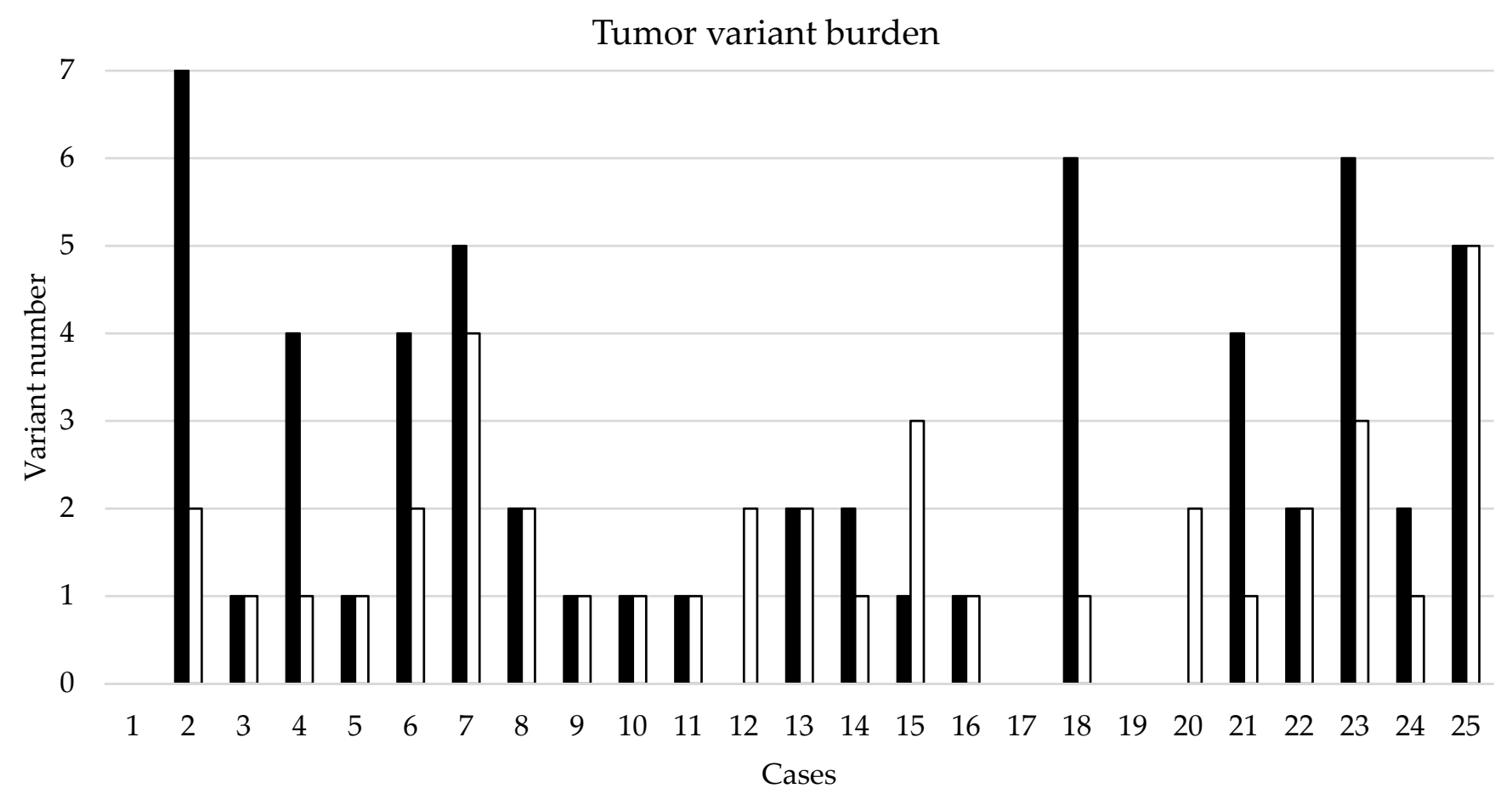

Tissue biopsy TVB $\quad \square$ Liquid biopsy TVB

Figure 3. Tumor variant burden in the 25 BTC cases. TVB was defined with the number of gene variants including SNPs above $2 \%$ VAF.

When comparing average TVB of the three BTC subtypes, no significant difference was identified (tissue vs. LB in IHCC: 2.1 vs. 1.6, $p=0.3612$; tissue vs. LB in EHCC: 1.4 vs. 0.8, $p=0.6374$; tissue vs. LB in GBC: 3.8 vs. $2.4, p=0.2371$ ). No significant difference was observed, comparing tissue biopsy-derived TVB (IHCC vs. EHCC: $p=0.5166$; IHCC vs. GBC: $p=0.1154$, EHCC vs. GBC: $p=0.1281$ ) and LB-derived TVB (IHCC vs. EHCC: $p=0.122$; IHCC vs. GBC: $p=0.2034$, EHCC vs. GBC: $p=0.0922)$, as well.

SNVs were detected in $21 / 25$ patients $(84 \%)$, while in cases $1,5,17$, and 19 , nucleotide aberration was not identified by our method. In two cases, only the LB sample was informative for molecular analysis (tissue biopsy insufficient for molecular analysis, case 12 and 20). Detected SNVs and their clinical significance were presented in Table 3.

Table 3. Detected single-nucleotide variants.

\begin{tabular}{|c|c|c|c|c|c|c|}
\hline Cases & Gene & Nucleotide Change & Amino Acid Change & VAF (\%) Tissue & VAF (\%) LB & Clinical Significance \\
\hline \multirow{6}{*}{2} & $K R A S$ & c. $407 \mathrm{G}>\mathrm{A}$ & p.Ser136Asn & 50 & 21.3 & pathogenic \\
\hline & IDH1 & c. $395 \mathrm{G}>\mathrm{T}$ & p.Arg132Leu & 25 & 19.6 & pathogenic \\
\hline & PTEN & c. $925 \mathrm{G}>\mathrm{A}$ & p.Ala309Thr & 20 & 15.2 & benign \\
\hline & FBXW7 & c. $239 \mathrm{C}>\mathrm{T}$ & p.Thr80Ile & 6 & 0 & likely pathogenic \\
\hline & IDH2 & c. $332 \mathrm{G}>\mathrm{A}$ & p.Gly111Asp & 4.6 & 0 & likely benign \\
\hline & $\mathrm{CDH1}$ & c. $596 \mathrm{C}>\mathrm{T}$ & p.Thr199Ile & 4.3 & 0 & likely benign \\
\hline
\end{tabular}


Table 3. Cont.

\begin{tabular}{|c|c|c|c|c|c|c|}
\hline Cases & Gene & Nucleotide Change & Amino Acid Change & VAF (\%) Tissue & VAF (\%) LB & Clinical Significance \\
\hline 3 & PIK3CA & c. $1634 \mathrm{~A}>\mathrm{C}$ & p.Glu545Ala & 3 & 3 & pathogenic \\
\hline \multirow{3}{*}{6} & FOXL2 & c. $536 C>G$ & p.Ala179Gly & 57.6 & 15.2 & benign \\
\hline & $A P C$ & c. $7504 \mathrm{G}>\mathrm{A}$ & p.Gly2502Ser & 49.5 & 6.7 & likely pathogenic \\
\hline & PIK3CA & c. $1634 \mathrm{~A}>\mathrm{C}$ & p.Glu545Ala & 3.8 & 0 & pathogenic \\
\hline \multirow{3}{*}{7} & $J A K 3$ & c. $2164 \mathrm{G}>\mathrm{A}$ & p.Val722Ile & 45.5 & 6.9 & benign \\
\hline & TP53 & c. $518 \mathrm{~T}>\mathrm{C}$ & p.Val173Ala & 13.2 & 4.9 & pathogenic \\
\hline & KRAS & c. $35 \mathrm{G}>\mathrm{T}$ & p.Gly12Val & 12.8 & 3.6 & pathogenic \\
\hline 8 & TP53 & c. $460 \mathrm{G}>\mathrm{A}$ & p.Gly154Ser & 4 & 2.1 & pathogenic \\
\hline 9 & FGFR2 & c. $827 \mathrm{~T}>\mathrm{G}$ & p.Phe276Cys & 37 & 6.3 & pathogenic \\
\hline 10 & IDH2 & c. $515 \mathrm{G}>\mathrm{T}$ & p.Arg172Met & 24.3 & 21.5 & pathogenic \\
\hline 11 & IDH2 & c. $359 \mathrm{G}>\mathrm{A}$ & p.Arg120Lys & 27.6 & 8.4 & pathogenic \\
\hline \multirow{2}{*}{12} & KDR & c. $1444 \mathrm{~T}>\mathrm{C}$ & p.Cys482Arg & \multirow{2}{*}{ Low tumor cell ratio } & 47.2 & pathogenic \\
\hline & STK11 & c. $1189 \mathrm{G}>\mathrm{A}$ & p.Ala397Thr & & 46.3 & benign \\
\hline \multirow{2}{*}{13} & TP53 & c. $536 \mathrm{~A}>\mathrm{G}$ & p.His179Arg & 62.6 & 5.6 & pathogenic \\
\hline & $\mathrm{IDH} 2$ & c. $359 \mathrm{G}>\mathrm{A}$ & p.Arg120Lys & 30.2 & 4.2 & pathogenic \\
\hline 14 & $M E T$ & c. $2975 \mathrm{C}>\mathrm{T}$ & p.Thr992Ile & 0 & 45.8 & pathogenic \\
\hline \multirow{3}{*}{15} & CSF1R & c. $2916 \mathrm{C}>\mathrm{G}$ & p.Cys972Trp & 0 & 7.9 & benign \\
\hline & $R E T$ & c. $1946 \mathrm{C}>\mathrm{T}$ & p.Ser649Leu & 0 & 7.8 & likely pathogenic \\
\hline & FOXL2 & c. $536 \mathrm{C}>\mathrm{G}$ & p.Ala179Gly & 61.6 & 4.5 & benign \\
\hline 16 & TP53 & c.796G $>\mathrm{T}$ & p.Gly266Ter & 29.2 & 5.2 & pathogenic \\
\hline \multirow{4}{*}{18} & MET & c. $1124 \mathrm{~A}>\mathrm{G}$ & p.Asn375Ser & 59.7 & 48.21 & pathogenic \\
\hline & HRAS & c. $182 A>G$ & p.Gln61Arg & 33.3 & 0 & pathogenic \\
\hline & PIK3CA & c. $40 \mathrm{C}>\mathrm{A}$ & p.His14Asn & 7.2 & 0 & likely benign \\
\hline & PIK3CA & c. $1634 \mathrm{~A}>\mathrm{C}$ & p.Glu545Ala & 4.6 & 0 & pathogenic \\
\hline \multirow{2}{*}{20} & FOXL2 & c. $536 \mathrm{C}>\mathrm{G}$ & p.Ala179Gly & \multirow{2}{*}{ Low tumor cell ratio } & 67.5 & benign \\
\hline & PIK3CA & c. $1571 G>A$ & p.Arg524Lys & & 7.7 & pathogenic \\
\hline 21 & $N R A S$ & c. $104 \mathrm{C}>\mathrm{T}$ & p.Thr35Ile & 8.3 & 2.6 & benign \\
\hline \multirow{2}{*}{22} & CDKN2A & c. $442 \mathrm{G}>\mathrm{A}$ & p.Ala148Thr & 57.8 & 52 & benign \\
\hline & $E G F R$ & c. $2543 \mathrm{C}>\mathrm{T}$ & p.Pro848Leu & 41 & 39 & likely benign \\
\hline \multirow{5}{*}{23} & CDKN2A & c. $442 \mathrm{G}>\mathrm{A}$ & p.Ala148Thr & 49 & 52.8 & benign \\
\hline & $\mathrm{CDH1}$ & c. $2474 \mathrm{C}>\mathrm{T}$ & p.Pro825Leu & 46 & 50.7 & pathogenic \\
\hline & HNF1A & c.862delG & p.Pro291GlnfsTer51 & 5.2 & 5.14 & likely pathogenic \\
\hline & TP53 & c. $325 \mathrm{~T}>\mathrm{G}$ & p.Phe109Val & 4.9 & 0 & pathogenic \\
\hline & PTEN & c. $802 \mathrm{G}>\mathrm{T}$ & p.Asp268Tyr & 4.6 & 0 & pathogenic \\
\hline 24 & STK11 & c.842del & p.Pro281ArgfsTer6 & 6.3 & 0 & pathogenic \\
\hline \multirow{6}{*}{25} & MLH1 & c. $1321 G>A$ & p.Ala441Thr & 46.2 & 49.1 & pathogenic \\
\hline & TP53 & c.707_711del & p.Tyr236LeufsTer2 & 12.1 & 3.4 & likely pathogenic \\
\hline & PIK3CA & c. $1571 G>A$ & p.Arg524Lys & 11.3 & 5.3 & pathogenic \\
\hline & CTNNB1 & c. $133 \mathrm{~T}>\mathrm{C}$ & p.Ser45Pro & 11.2 & 2.4 & pathogenic \\
\hline & $K R A S$ & c. $34 \mathrm{G}>\mathrm{T}$ & p.Gly12Cys & 6.6 & 0 & pathogenic \\
\hline & FOXL2 & c.743T $>C$ & p.Leu248Pro & 0 & 3.1 & likely pathogenic \\
\hline
\end{tabular}

NGS on samples originating from tissue biopsy and matched liquid biopsy was performed.

The DNA VAF of tissue biopsy and LB were greatly varying with the average of $21.8 \%$ (range: $3.0-62.6$ ) and $13.32 \%$ (range: $2.1-67.5$ ), respectively. Pathogenic variations were proven at a rate of $17 / 25(68 \%)$ and presented in some of the most usually affected 
genes in BTCs, such as FGFR2, IDH1, IDH2, KRAS, and TP53, and most of them could be identified in matched LB-originated cfDNA. Pathogenic SNVs were referred to as mutations ( $n=28$ in the 25 patients), while benign aberrations were considered neutral $(n=15)$.

Twenty-five genes were affected in the BTC patients. FOXL2, PIK3CA, and TP53 SNVs have emerged in all BTC subgroups, while CDH1, KRAS, PTEN, and STK11 alteration was found in the IHCC and GBC cases, as well. MET aberration was proved in IHCC and EHCC group, but HRAS c.182A>G (p.Gln61Arg) variance was detected only in one EHCC sample. Several other molecular abnormalities were identified in IHCC and GBC. The genetically aberrant gene distribution of the three subtypes of BTCs was presented in Figure 4.

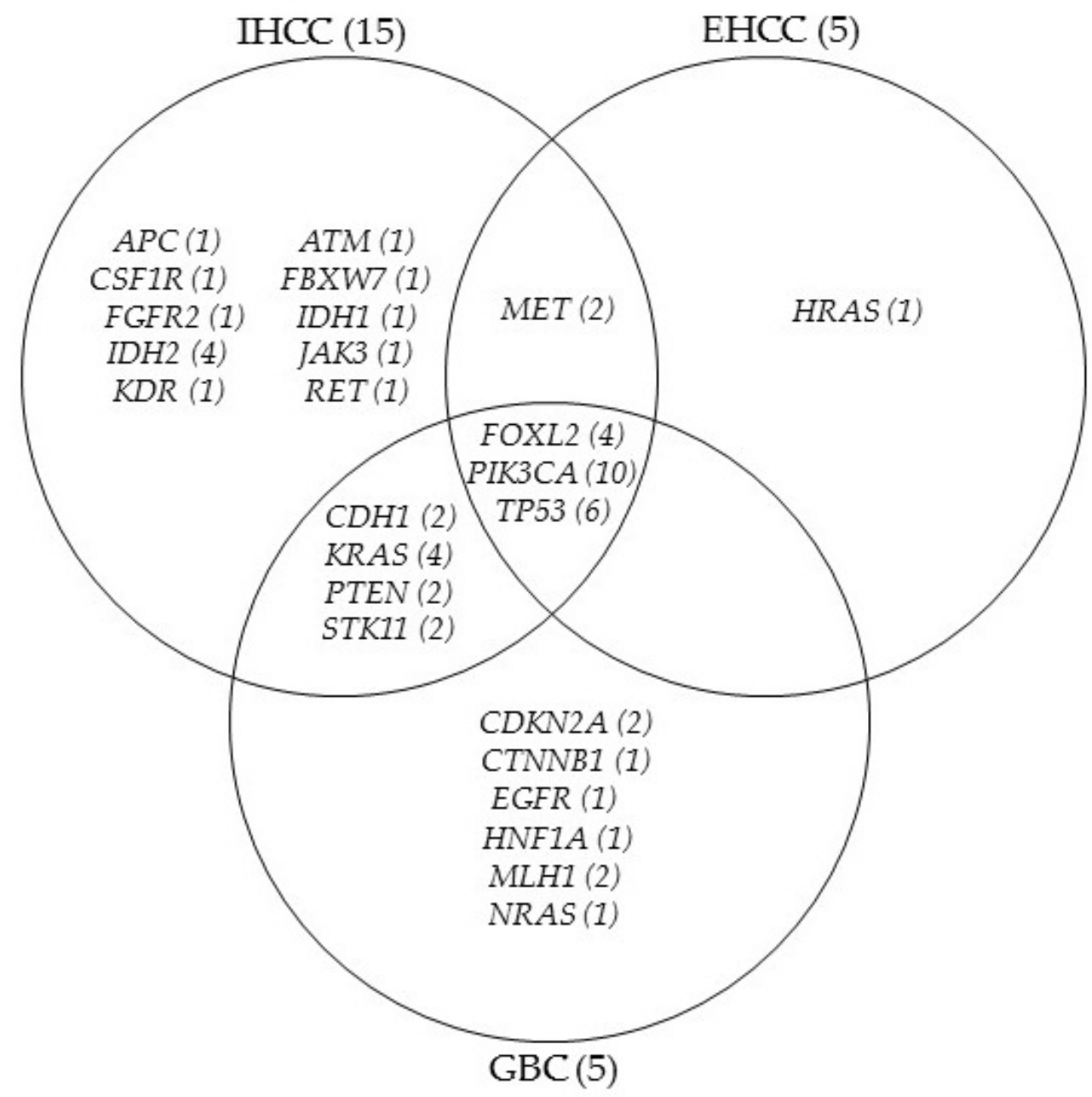

Figure 4. Genetically aberrant gene distribution of the three subtypes of BTCs. IHCC (15): intrahepatic cholangiocarcinoma, EHCC (5): extrahepatic cholangiocarcinoma, GBC (5): gallbladder carcinoma (case number are in bracket). Gene aberration frequencies are presented in the bracket after gene symbols.

\section{Discussion}

BTC is a rare malignancy with a distinctly poor prognosis and limited therapeutic options, so it would be essential to understand the molecular pathogenesis and find a specific molecular target for achieving an effective treatment [1,2]. In the area of precision oncology, not only obtaining an individual possibility of chemotherapy, but also early diagnosis is important for the oncological management of BTC patients. For this purpose, our prospective study focuses on the comprehensive genetic analysis of molecular aberrations of the background in cholangiocarcinoma.

Accessing genetic alterations, therefore, is essential for the diagnosis, management, and selection of targeted therapies, although sampling tumor tissue, when possible, is often risky and difficult to carry out. Compared to the traditional tissue biopsy, LB is not 
invasive and can be repeated as the potential substitute method for gaining information about the real-time molecular aberration background. The use of peripheral blood LB has been introduced in the early molecular diagnosis of several malignancies, such as lung and colorectal adenocarcinomas, and melanomas, as well [19,22-24]. More recently, LB application to detect aberrant gene fusions has been published for the diagnosis of aggressive lymphoma genotyping [25]. Little information was found about the utility of LB to identify diagnostic and prognostic biomarkers in BTCs [26], so for this reason we aimed to quantify and analyze cfDNA management of patients affected by BTC. No published data is available for the association between tumor mass and LB nucleic acid quantity. When determining the ETV in our study, a statistically significant correlation was proved between the tumor volume and peripheral blood plasma cfDNA concentration. The larger tumor mass, calculated based on recent in vivo CT/MR scans, was associated with higher cfDNA yield, as well. In cases with metastasis (case 2, 3, 7, 13, and 14), the metastatic tumor volume was added to ETV. LB was performed the mean 24 months after tissue biopsy (IHCC and EHCC cases), in these cases, ETV was not significantly different from the initial imaging scans. In GBC cases, where surgical resection was carried out, low cfDNA concentration was measured in the PB plasma.

Tumor Mutation Burden (TMB) is a relevant type of specific biomarker, which was introduced as a general molecular diagnostic feature to predict response to immunotherapy in a broad scale of malignancies [27]. TMB is calculated as the number of variants per Mbp genomic DNA isolated from the neoplastic tissue sample. There is very limited literature available on TMB data in association with BTCs [28]. To address this issue in our study, we calculated TVB for every case, not only for the tissue, but also in LB samples. TVB was defined in this study as the number of all gene variants including SNPs above $2 \%$ VAF. High TVB was determined to produce targeted NGS libraries of 660 regions of interest across 67 genes. Comparing tumor tissue and LB results, similar TVB was observed in most of the cases. No significant difference was identified when analyzing the average tissue biopsy-derived and LB-derived TVB of the three BTC subtypes. A total of 45 alterations were found in tissue and from these, 28 emerged in the plasma (62.2\%). Controversially, four SNVs were identified only in the plasma, while in two cases, only the LB sample was informative for molecular analysis (in case 12 and 20, four variants were detected in cfDNA).

Recent reports described gene aberrations related to the individual BTC subtypes that potentially contribute to different cholangiocarcinoma pathogenesis [3,16-18]. In our study, SNVs were identified in twenty-one patients and the results broadly reflected the differences in the three molecular categories described in the literature [16-18] (Figure 4). The highest number of the affected genes $(n=18)$ was found in the IHCC type, but eight of them were shared with either EHCC or GBC types. In the EHCC group, only five gene variants could be identified, four of them were common with the other subtypes, while one (HRAS) variant occurred exclusively in this subtype. Out of the total 13 gene alterations, six were identified in GBC, which has not emerged in the other two groups. CDH1, KRAS, PTEN, MET, and STK11 alterations were described in association with IHCC and GBC in some publications [29-31]. FOXL2, PIK3CA, and TP53 variants were found in all three BTC histologic subtypes. According to the literature data, these variations are most often, but not exclusively detected in the EHCC patients, while their occurrence in IHCC samples is associated with an unfavorable prognosis [16,32,33].

One of the most common gene mutations in cholangiocarcinoma is affecting the IDH1/IDH2 gene (encoding isocitrate dehydrogenase isotypes), which is characteristic of the IHCC category $[1,3,16]$. A similar finding was provided by our study, as it was detected only in IHCC patients with a frequency of $4 / 15$ (case 2, 10, 11, and 13). In case 2, IDH1 and IDH2 variants emerged at the same time. FGFR2 point mutation is one other major gene alteration in IHCC [29-31], which was found in a single case with pathogenic clinical significance (case 9).

Variants were further categorized according to their clinical significance as determined by the COSMIC and Clinvar databases. Pathogenic mutations were detected in 17 of 
25 patients (68\%). The most common affected genes in BTCs, such as FGFR2, IDH1, IDH2, $K R A S$, and TP53 presented with pathogenic variants in our study population, and most of them could be identified in matched LB originated cfDNA, as well. KRAS, BRAF mutations, overexpression of EGFR, HER2, activation of oncogenic signaling pathways, DNA amplification, and deletions are associated with poor outcomes [34,35].

Detected pathogen variants were cross-checked in the OncoKB database for therapeutic possibilities. Optional oncotherapeutic agent was found in only four cases, one was specific for BTC patients. Ivosidenib for IDH1 p.Arg132Leu aberration (case 2) was the FDA's approved therapeutic drug for the management of advanced cholangiocarcinoma patients [36,37]. AZD4547, BGJ398, Debio1347, and Erdafitinib for all oncogenic FGFR2 mutations, Tipifarnib for HRAS aberrations, and AZD8186, and GSK2636771 for PTEN point mutations were not specified in the FDA's approved packaging label for BTC therapy (off-label drug).

BTCs are extraordinary rare malignancies and diagnostic sampling is frequently extremely difficult due to the special anatomical localization, partial tissue involvement, or minimal amounts of the tissue sample. Thus, our study design was also faced with such issues, which could be partially overcome when applying tumor tissue and LB-based sequencing. The major limitation of our study is the low case number originating from a single institution. The use of an NGS gene panel targeting 67 genes appeared highly effective in both sample types, although did not fully cover all affected genes described in BTCs. The presence of copy number variations (ERBB2) and fusions (FGFR2, NTRK) are important in BTC and are not covered in the assay, as well. LB was carried out only one time to control the chemotherapy effectiveness and no clinical follow-up was performed because most of the patients were treated with similar onco-chemotherapeutic agents.

\section{Conclusions}

Tumor-derived cfDNA in BTCs also allows the effective monitoring of the molecular genetic profile and the response to chemotherapy. The use of LB is a favorable solution because repeated invasive sampling can be avoided. We assume that the efficacy of the LB-based sequencing is increasing with the progression and cfDNA release of the tumor. In the present series, we were able to demonstrate clinically relevant SNVs from the tissue and matched LB samples of BTC patients. Our prospective study demonstrates a minimally invasive testing approach to identify molecular genetic alterations in cholangiocarcinoma and gallbladder cancers. Clinical applications of cfDNA reflect by capturing the outstanding spatial tumor heterogeneity and guarantee novel aspects for precision oncology treatment.

Author Contributions: S.L.C., data curation, formal analysis, investigation, methodology, project administration, and writing — original draft preparation; J.B., investigation, methodology, and writingoriginal draft preparation; G.V., data curation, formal analysis, methodology, and visualization; A.Á. and C.A., data curation, and investigation; G.M., conceptualization, formal analysis, funding acquisition, investigation, resources, supervision, and writing - review and editing; A.M., conceptualization, data curation, formal analysis, investigation, methodology, project administration, supervision, visualization, and writing - review and editing. All authors have read and agreed to the published version of the manuscript.

Funding: This research received no external funding.

Institutional Review Board Statement: The study was conducted according to the guidelines of the Declaration of Helsinki, and approved by the Hungarian Medical Research Council (protocol code 60355-2/2016/EKU and 4648-6/2018/EÜIG).

Informed Consent Statement: Informed consent was obtained from all subjects involved in the study.

Data Availability Statement: The data presented in this study are available on request from the corresponding author. The data are not publicly available to protect the rights of patients.

Conflicts of Interest: The authors declare no conflict of interest. 


\section{References}

1. Noel, M.S.; Hezel, A.F. New and Emerging Treatment Options for Biliary Tract Cancer. OncoTargets Ther. 2013, 6, 1545-1552. [CrossRef]

2. Weinberg, B.A.; Xiu, J.; Lindberg, M.R.; Shields, A.F.; Hwang, J.J.; Poorman, K.; Salem, M.E.; Pishvaian, M.J.; Holcombe, R.F.; Marshall, J.L.; et al. Molecular Profiling of Biliary Cancers Reveals Distinct Molecular Alterations and Potential Therapeutic Targets. J. Gastrointest. Oncol. 2019, 10, 652-662. [CrossRef]

3. Putra, J.; de Abreu, F.B.; Peterson, J.D.; Pipas, J.M.; Mody, K.; Amos, C.I.; Tsongalis, G.J.; Suriawinata, A.A. Molecular Profiling of Intrahepatic and Extrahepatic Cholangiocarcinoma Using next Generation Sequencing. Exp. Mol. Pathol. 2015, 99, 240-244. [CrossRef]

4. Horgan, A.M.; Amir, E.; Walter, T.; Knox, J.J. Adjuvant Therapy in the Treatment of Biliary Tract Cancer: A Systematic Review and Meta-Analysis. J. Clin. Oncol. 2012, 30, 1934-1940. [CrossRef]

5. Ma, N.; Cheng, H.; Qin, B.; Zhong, R.; Wang, B. Adjuvant Therapy in the Treatment of Gallbladder Cancer: A Meta-Analysis. BMC Cancer 2015, 15, 615. [CrossRef]

6. Lamarca, A.; Hubner, R.A.; David Ryder, W.; Valle, J.W. Second-Line Chemotherapy in Advanced Biliary Cancer: A Systematic Review. Ann. Oncol. 2014, 25, 2328-2338. [CrossRef]

7. Ben-Josef, E.; Guthrie, K.A.; El-Khoueiry, A.B.; Corless, C.L.; Zalupski, M.M.; Lowy, A.M.; Thomas, C.R.; Alberts, S.R.; Dawson, L.A.; Micetich, K.C.; et al. SWOG S0809: A Phase II Intergroup Trial of Adjuvant Capecitabine and Gemcitabine Followed by Radiotherapy and Concurrent Capecitabine in Extrahepatic Cholangiocarcinoma and Gallbladder Carcinoma. J. Clin. Oncol. 2015, 33, 2617-2622. [CrossRef]

8. Primrose, J.N.; Fox, R.P.; Palmer, D.H.; Malik, H.Z.; Prasad, R.; Mirza, D.; Anthony, A.; Corrie, P.; Falk, S.; Finch-Jones, M.; et al. Capecitabine Compared with Observation in Resected Biliary Tract Cancer (BILCAP): A Randomised, Controlled, Multicentre, Phase 3 Study. Lancet Oncol. 2019, 20, 663-673. [CrossRef]

9. Ebata, T.; Hirano, S.; Konishi, M.; Uesaka, K.; Tsuchiya, Y.; Ohtsuka, M.; Kaneoka, Y.; Yamamoto, M.; Ambo, Y.; Shimizu, Y.; et al. Randomized Clinical Trial of Adjuvant Gemcitabine Chemotherapy versus Observation in Resected Bile Duct Cancer. Br. J. Surg. 2018, 105, 192-202. [CrossRef]

10. Edeline, J.; Benabdelghani, M.; Bertaut, A.; Watelet, J.; Hammel, P.; Joly, J.-P.; Boudjema, K.; Fartoux, L.; Bouhier-Leporrier, K.; Jouve, J.-L.; et al. Gemcitabine and Oxaliplatin Chemotherapy or Surveillance in Resected Biliary Tract Cancer (PRODIGE 12-ACCORD 18-UNICANCER GI): A Randomized Phase III Study. J. Clin. Oncol. 2019, 37, 658-667. [CrossRef]

11. Lamarca, A.; Palmer, D.H.; Wasan, H.S.; Ross, P.J.; Ma, Y.T.; Arora, A.; Falk, S.; Gillmore, R.; Wadsley, J.; Patel, K.; et al. Second-Line FOLFOX Chemotherapy versus Active Symptom Control for Advanced Biliary Tract Cancer (ABC-06): A Phase 3, Open-Label, Randomised, Controlled Trial. Lancet Oncol. 2021, 22, 690-701. [CrossRef]

12. Tamai, K.; Nakamura, M.; Mizuma, M.; Mochizuki, M.; Yokoyama, M.; Endo, H.; Yamaguchi, K.; Nakagawa, T.; Shiina, M.; Unno, M.; et al. Suppressive Expression of CD274 Increases Tumorigenesis and Cancer Stem Cell Phenotypes in Cholangiocarcinoma. Cancer Sci. 2014, 105, 667-674. [CrossRef]

13. Fontugne, J.; Augustin, J.; Pujals, A.; Compagnon, P.; Rousseau, B.; Luciani, A.; Tournigand, C.; Cherqui, D.; Azoulay, D.; Pawlotsky, J.-M.; et al. PD-L1 Expression in Perihilar and Intrahepatic Cholangiocarcinoma. Oncotarget 2017, 8, $24644-24651$. [CrossRef]

14. Mody, K.; Starr, J.; Saul, M.; Poorman, K.; Weinberg, B.A.; Salem, M.E.; VanderWalde, A.; Shields, A.F. Patterns and Genomic Correlates of PD-L1 Expression in Patients with Biliary Tract Cancers. J. Gastrointest. Oncol. 2019, 10, 1099-1109. [CrossRef]

15. Cardinale, V.; Carpino, G.; Reid, L.; Gaudio, E.; Alvaro, D. Multiple Cells of Origin in Cholangiocarcinoma Underlie Biological, Epidemiological and Clinical Heterogeneity. World J. Gastrointest. Oncol. 2012, 4, 94-102. [CrossRef]

16. Churi, C.R.; Shroff, R.; Wang, Y.; Rashid, A.; Kang, H.C.; Weatherly, J.; Zuo, M.; Zinner, R.; Hong, D.; Meric-Bernstam, F.; et al. Mutation Profiling in Cholangiocarcinoma: Prognostic and Therapeutic Implications. PLoS ONE 2014, 9, e115383. [CrossRef]

17. Nakamura, H.; Arai, Y.; Totoki, Y.; Shirota, T.; Elzawahry, A.; Kato, M.; Hama, N.; Hosoda, F.; Urushidate, T.; Ohashi, S.; et al. Genomic Spectra of Biliary Tract Cancer. Nat. Genet 2015, 47, 1003-1010. [CrossRef]

18. Brandi, G.; Farioli, A.; Astolfi, A.; Biasco, G.; Tavolari, S. Genetic Heterogeneity in Cholangiocarcinoma: A Major Challenge for Targeted Therapies. Oncotarget 2015, 6, 14744-14753. [CrossRef]

19. Méhes, G. Liquid Biopsy for Predictive Mutational Profiling of Solid Cancer: The Pathologist's Perspective. J. Biotechnol. 2019, 297, 66-70. [CrossRef]

20. Rizzo, A.; Ricci, A.D.; Tavolari, S.; Brandi, G. Circulating Tumor DNA in Biliary Tract Cancer: Current Evidence and Future Perspectives. Cancer Genom. Proteom. 2020, 17, 441-452. [CrossRef]

21. Han, J.-Y.; Ahn, K.S.; Kim, T.-S.; Kim, Y.H.; Cho, K.B.; Shin, D.W.; Baek, W.-K.; Suh, S.-I.; Jang, B.-C.; Kang, K.J. Liquid Biopsy from Bile-Circulating Tumor DNA in Patients with Biliary Tract Cancer. Cancers 2021, 13, 4581. [CrossRef]

22. Rachiglio, A.M.; Esposito Abate, R.; Sacco, A.; Pasquale, R.; Fenizia, F.; Lambiase, M.; Morabito, A.; Montanino, A.; Rocco, G.; Romano, C.; et al. Limits and Potential of Targeted Sequencing Analysis of Liquid Biopsy in Patients with Lung and Colon Carcinoma. Oncotarget 2016, 7, 66595-66605. [CrossRef] [PubMed]

23. Mack, P.C.; Banks, K.C.; Espenschied, C.R.; Burich, R.A.; Zill, O.A.; Lee, C.E.; Riess, J.W.; Mortimer, S.A.; Talasaz, A.; Lanman, R.B.; et al. Spectrum of Driver Mutations and Clinical Impact of Circulating Tumor DNA Analysis in Non-Small Cell Lung Cancer: Analysis of over 8000 Cases. Cancer 2020, 126, 3219-3228. [CrossRef] [PubMed] 
24. Gaiser, M.R.; von Bubnoff, N.; Gebhardt, C.; Utikal, J.S. Liquid Biopsy to Monitor Melanoma Patients. J. Dtsch. Dermatol. Ges. 2018, 16, 405-414. [CrossRef] [PubMed]

25. Mokánszki, A.; Bicskó, R.; Gergely, L.; Méhes, G. Cell-Free Total Nucleic Acid-Based Genotyping of Aggressive Lymphoma: Comprehensive Analysis of Gene Fusions and Nucleotide Variants by Next-Generation Sequencing. Cancers 2021, $13,3032$. [CrossRef]

26. Rompianesi, G.; Di Martino, M.; Gordon-Weeks, A.; Montalti, R.; Troisi, R. Liquid Biopsy in Cholangiocarcinoma: Current Status and Future Perspectives. World J. Gastrointest. Oncol. 2021, 13, 332-350. [CrossRef]

27. Samstein, R.M.; Lee, C.-H.; Shoushtari, A.N.; Hellmann, M.D.; Shen, R.; Janjigian, Y.Y.; Barron, D.A.; Zehir, A.; Jordan, E.J.; Omuro, A.; et al. Tumor Mutational Load Predicts Survival after Immunotherapy across Multiple Cancer Types. Nat. Genet. 2019, 51, 202-206. [CrossRef] [PubMed]

28. Chen, X.; Wu, X.; Wu, H.; Gu, Y.; Shao, Y.; Shao, Q.; Zhu, F.; Li, X.; Qian, X.; Hu, J.; et al. Camrelizumab plus Gemcitabine and Oxaliplatin (GEMOX) in Patients with Advanced Biliary Tract Cancer: A Single-Arm, Open-Label, Phase II Trial. J. Immunother. Cancer 2020, 8, e001240. [CrossRef]

29. Jusakul, A.; Cutcutache, I.; Yong, C.H.; Lim, J.Q.; Huang, M.N.; Padmanabhan, N.; Nellore, V.; Kongpetch, S.; Ng, A.W.T.; $\mathrm{Ng}$, L.M.; et al. Whole-Genome and Epigenomic Landscapes of Etiologically Distinct Subtypes of Cholangiocarcinoma. Cancer Discov. 2017, 7, 1116-1135. [CrossRef]

30. Mertens, J.C.; Rizvi, S.; Gores, G.J. Targeting Cholangiocarcinoma. Biochim. Biophys. Acta Mol. Basis Dis. 2018, 1864, 1454-1460. [CrossRef]

31. Rizvi, S.; Khan, S.A.; Hallemeier, C.L.; Kelley, R.K.; Gores, G.J. Cholangiocarcinoma-Evolving Concepts and Therapeutic Strategies. Nat. Rev. Clin. Oncol. 2018, 15, 95-111. [CrossRef]

32. Montal, R.; Sia, D.; Montironi, C.; Leow, W.Q.; Esteban-Fabró, R.; Pinyol, R.; Torres-Martin, M.; Bassaganyas, L.; Moeini, A.; Peix, J.; et al. Molecular Classification and Therapeutic Targets in Extrahepatic Cholangiocarcinoma. J. Hepatol. 2020, 73, 315-327. [CrossRef]

33. Lendvai, G.; Szekerczés, T.; Illyés, I.; Dóra, R.; Kontsek, E.; Gógl, A.; Kiss, A.; Werling, K.; Kovalszky, I.; Schaff, Z.; et al Cholangiocarcinoma: Classification, Histopathology and Molecular Carcinogenesis. Pathol. Oncol. Res. 2020, 26, 3-15. [CrossRef]

34. Andersen, J.B.; Spee, B.; Blechacz, B.R.; Avital, I.; Komuta, M.; Barbour, A.; Conner, E.A.; Gillen, M.C.; Roskams, T.; Roberts, L.R.; et al. Genomic and Genetic Characterization of Cholangiocarcinoma Identifies Therapeutic Targets for Tyrosine Kinase Inhibitors. Gastroenterology 2012, 142, 1021-1031.e15. [CrossRef] [PubMed]

35. Sia, D.; Hoshida, Y.; Villanueva, A.; Roayaie, S.; Ferrer, J.; Tabak, B.; Peix, J.; Sole, M.; Tovar, V.; Alsinet, C.; et al. Integrative Molecular Analysis of Intrahepatic Cholangiocarcinoma Reveals 2 Classes That Have Different Outcomes. Gastroenterology 2013, 144, 829-840. [CrossRef] [PubMed]

36. Abou-Alfa, G.K.; Macarulla, T.; Javle, M.M.; Kelley, R.K.; Lubner, S.J.; Adeva, J.; Cleary, J.M.; Catenacci, D.V.; Borad, M.J.; Bridgewater, J.; et al. Ivosidenib in IDH1-Mutant, Chemotherapy-Refractory Cholangiocarcinoma (ClarIDHy): A Multicentre, Randomised, Double-Blind, Placebo-Controlled, Phase 3 Study. Lancet Oncol. 2020, 21, 796-807. [CrossRef]

37. Zhu, A.X.; Macarulla, T.; Javle, M.M.; Kelley, R.K.; Lubner, S.J.; Adeva, J.; Cleary, J.M.; Catenacci, D.V.T.; Borad, M.J.; Bridgewater, J.A.; et al. Final Overall Survival Efficacy Results of Ivosidenib for Patients With Advanced Cholangiocarcinoma with IDH1 Mutation: The Phase 3 Randomized Clinical ClarIDHy Trial. JAMA Oncol. 2021, 7, 1669-1677. [CrossRef] [PubMed] 